\title{
Diagnostic Challenges inInfections related to Spinal Cord Injury
}

\author{
Murtaza Mustafa $^{1}$, HM.Iftikhar ${ }^{2}$, H.Firdaus ${ }^{3}$, EM.Illzam ${ }^{4}$, S.Hamed ${ }^{5}$, \\ B. Almohtafar ${ }^{6}$ \\ ${ }^{1,3,5}$, Faculty of Medicine And Health Sciences, Universitymalaysia,Sabah,Kota Kinabalu,Sabah,Malaysia \\ ${ }^{2}$ Department of Orthopedic, Management and Science University, Selangor, Malaysia \\ ${ }^{4}$ Clinic Family Planning Association,Kota Kinabalu,Sabah,Malaysia \\ ${ }^{6}$ Departments of Surgery, UCSI University Terengganu Campus, Terengganu, Malaysia.
}

\begin{abstract}
Spinal cord injury (SCI) is an ancient disease, described by Hippocrates and traction techniques were recommended to straighten the spine. Motor vehicle accidents, falls, attempted suicide, gunshot wounds, military conflicts and support injuries are most common cause of SCIs. Most hospitalized SCI patients have nosocomial infection. SCI Patients may suffer from stress, malnutrition, renal failure; receive medications that impair immune responses. Frequent insertion of urologic, vascular, orthopedic, respiratory, gastrointestinal, and neurosurgical devices predispose to various prosthesis-related infections. Diagnostic challenges include inability to recognize the signs and symptoms of cord damage, and delay in diagnosing spinal epidural abscess. Treatment of infection e.g., two opposing factors resulting from changes in body composition in following SCI can alter the disposition of systematically administered antibiotics, such as vancomycin and aminoglycosides. Frequently diagnosed infections in SCI patients are urinary tract infection (UTI), pneumonia, pressure sores, osteomyelitis, infection due to multi-resistant organisms, and intra-abdominal infection. SCI patients are at high risk for Fournier gangrene that affects the perineal and genital regions and usually results from polymicrobial infection.Common pathogens are Staphylococcus aureus, including methicillin resistant Staphylococcus aureus(MRSA),Streptococci, vancomycin resistant Enterococcus(VRE)gram-negative bacilli including ESBL producing Klebsiella pneumoniae and Escherichia coli. Earlydiagnosis, gram negative coverage when initiating empirical antibiotic therapy has lower mortality and better outcomes.
\end{abstract}

Keywords: Spinal cord injury, Urinary tract infection,Pressuresores,Diagnostic challenges.

\section{Introduction}

Much has changed since ancient Egyptian physician viewed spinal injury "an ailment not be treated"[1,].More than 250,000 Americans currently suffer from the consequence of spinal cord injury(SCI) and,with an annual incidence of 40 cases per million population, over 11,000 new cases of SCI occur in the United States each year[2,].About one third of SCI patients develop infection during hospitalization, with an overall incidence of 35 episodes of hospital acquired infection/1000 hospital days[3].SCI are most often caused by physical trauma[4].In the US, Motor Vehicle accidents are most common cause of SCIs; second are falls, then violence such as gunshot wounds, then sports injuries[5].Other causes of SCIs include, attempted suicide, military conflicts, and iatrogenic injury, caused by an improperly done medical procedures such as an injection into spinal column[6-8].In Malaysia motor vehicle accidents(66\%) and falls(28\%) are the main cause of SCIs[9].Most infections that occur in SCI patients also affect able-bodied patient, but the frequency and clinical characteristics of infections vary between those populations. Whereas urinary tract infections are the most common[10],pneumonia has the highest infection-associated mortality[11], and infections of pressure sores and underlying bone are probably most difficult to manage[12].Contributory factors to SClinfection include, frequent insertion of urologic, vascular, orthopedic, respiratory ,gastrointestinal, and neurosurgical devices predispose to various prosthesis-related infections[3].Infection frequently manifests differently in the SCI population than in able-bodied individuals. Altered or absent sensation constitute the single most impediments to the diagnosis of infection in this population [13].Treatment of infection SCI patients also poses special challenges. For example, two opposing factors resulting from changes in body composition following SCI can alter the disposition of systematically administered antibiotics, such as vancomycin and aminoglycosides 
$[14,15]$.The paper reviews the current literature, diagnostic challenges, and infections in patients with spinal cord injury.

\section{Historical Perspectives}

Spinal Injury has been known to be devastating for millennia, the ancient Egyptian Edwin Smith Payrus from $2500 \mathrm{BC}$, the first known description of the injury, says it is "not to be treated"[16].Hindu text dating back to $1800 \mathrm{BC}$ also mention SCI and described traction techniques to straighten the spine[16].Hippocrates described SCI in his Hippocratic Corpus and invented traction devices to straighten the dislocated vertebrae[17].But it was not until Aulus Cornelius Celsus,born 30 BC,noted that a cervical injury resulted in rapid death that spinal cord itself was implicated in the condition[16].In the second century AD the Greek physician Galen experimented on monkeys and reported that a horizontal cut through the spinal cord caused them to lose all sensation below the level of the cut [18].The seventh- century Greek physician Paul of Aegina described surgical techniques for treatment of broken vertebrae by removing the broken fragments, as well as surgery to relieve pressure on the spine[16].

In 1762 a surgeon named Andre Louis removed a bullet from the lumbar spine of a patient, who regained motion in legs[18].In 1829 the surgeon Gilpin performed a successful laminectomy that improved patient's sensation[19].However that SCI was untreatable remained predominant until the early 20th century[20].In the 1934 the mortality rate in the first two years after injury was over $80 \%$ mostly due to infections of the urinary tract and pressure sores[21].Improvement in care have accompanied by increased life expectancy of people with SCI; survival times have improved by $2000 \%$ since 1940 [22].

\section{Predisposing factors to infection}

SCI does not depress general host immunity. Uninfected individuals with SCI have a normal function of $\mathrm{T}$ and B lymphocytes. Although patients with SCI usually have higher levels of inflammatory markers, including C-reactive protein and cytokines such as interleukin- 6 and tumor necrosis factor- $\alpha$, than able- bodied cohorts [23,24],this difference can be attributed to undetected inflammation or occult infection.However,SCI patients may suffer from complicating conditions (including stress, malnutrition, and renal failure) or receive medications(e.g., high -dose glucocorticosteroids in acute setting of SCI) that can impair immune response to infection. More importantly, SCI patients possess unique factors that predispose them to infection of specific body organ systems.

For example, patients have a neurogenic bladder and suffer from frequent urinary tract infection attributed to urinary stasis and bladder catheterization. Urinary stasis greatly impairs the naturally protective mechanisms of the urinary tract, such as the washout effect of voiding and the phagocytic capacity of bladder epithelial cells. Even though some techniques of bladder catheterization are safer than others, none can be carried out without the potential risk of introducing organisms into the urinary tract. Both paralytic ileus and abnormal state of the consciousness caused by associated head injury illicit drug ingestion can predispose to aspiration pneumonia in the acute stage of SCI.In persons with cervical or high thoracic cord lesions, weakness of the diaphragmatic and intercostal muscles impairs the capacity to clear respiratory secretions. Skin breakdown in anesthetic areas, immobility, disuse-induced muscle atrophy, urinary leakage and fecal contamination predispose to infection $[23,24]$.

Patients with SCI generally have a higher rate of hospital - acquired infection (e.g.,urinary tract, blood stream, and musculoskeletal system) than other groups of patients[3].

\section{Diagnostic challenges}

A number of unique challenges can be encountered when attempting to establish diagnosis and provide treatment. Infection frequently manifests differently in the SCI patients than in other groups. For example,dysuria, frequency, and urgency, symptoms that are regularly present in able- bodied patients who suffer from urinary tract infection rarely exist in infected SCI patients. The diagnosis of perinephric abscess is particularly challenging in patients with high sensory levels who do not appreciate flank pain or tenderness [3,].The inability to recognize the signs and symptoms of cord damage contributes to the delay in diagnosing spinal epidural abscess below the level of injury [25].The diagnostic dilemma caused by the paucity of clinical 
findings can be heightened by the presence of neurogenic or referred pain that may not be related the infection. Furthermore, multiple infections occur concurrently in up to $20 \%$ of SCI patients. Even more problematic than identifying the source of infection is discerning whether fever is caused by an infection or noninfectious conditions that may closely mimic infections and cause almost on fifth of episodes of fever in SCI patients [26]. A diagnostic conundrum may exist when unique thermoregulatory and autonomic disturbances cause fever in SCI patients. Because of the imbalance between heat production and heat loss, patients with an SCI above T8 may not be able to maintain normal body temperature in response to heating or cooling (poikilothermia)[27].This phenomenon of altered thermoregulation is attributed to the loss of sweating and muscular activity below the spinal cord lesion. These factors may contribute to the occurrence of self-limited febrile episodes in SCI patients that resolve spontaneously within hours to days. However, neither alterations in environmental temperature nor changes in a subject's sweating and muscular activity may explain the occurrence of prolonged fever in recently injured quadriplegic patients who have no identifiable focus of infection [28].This unique syndrome, so- called quadriplegia fever, lasts weeks to months and is problematic because it may incite repeated evaluation of for infection and multiple courses of antibiotics, but no avail. Rarely, fever may occur in the contest of autonomic dysreflexia, a paroxamal syndrome characterized mainly by hypertension, sweating, facial flushing, and headache. Occasionally, bradycardia may also be present and can help differentiate febrile episodes of autonomic dysreflexia from infection. This type of autonomic hyperactivity is seen in patients with SCI above T 6 and is usually triggered by distention by viscera(bladder and rectum),cutaneous stimulation(e.g., ingrown toenails), or even infection[26].

Treatment of infection in SCI patients also poses special challenges. For example, two opposing factors resulting from changes in body composition in following SCI can alter the disposition of systematically administered antibiotics, such as vancomycin and aminoglycosides[14,15]. On one hand, patients with SCI have an expanded extracellular volume attributed to retention of extracellular water as subclinical edema and replacement of decreased skeletal muscle mass muscle mass by extracellular water. As a result, these patients have a larger weight adjusted volume of distribution of drugs and may require larger weight-adjusted loading and maintenance doses than able-bodied counterparts to achieve similar antibiotic concentrations. This potential effect on antibiotic concentration can be countered, at least in part by the frequent overestimation of creatinine clearance when using formulas that were originally devised for non-SCI individuals to predict creatinine clearance in patients with chronic SCI who have low serum creatinine levels [29].This misapplication of such formulas has prompted the evaluation of modified methods for proper administration of vancomycin and other antibiotics in the SCI population[26].

\section{Urinary tract Infection (UTI)}

\section{SCI Infection Syndrome}

This is the most common infection in patients with SCI and occurs at a rate of 2.5 episodes/patient/year. In patients with chronic indwelling bladder catheters (transurethral or suprapubic)., bacteremia is almost universal(culture of a randomly obtained urine sample is positive in almost $98 \%$ of cases)[7].

Togan and associates in a prospective study reported that $22 \%$ of patients develop UTI during the first 50 days and $20 \%$ of cases among chronic SCI[30].These SCI patients have a higher rate of bacteriuria than those who rely on intermittent bladder cauterization( $98 \%$ vs $70 \%)$.A longer interval between intermittent bladder catheterization may be associated with a higher incidence of bacteriuria. Although outpatient may find it more practical to use clean reusable rather than sterile catheters for intermittent bladder catheterization, there is conflicting evidence regarding the value of clean versus sterile bladder catheterization [31].

Most cases of UTI in SCI patients are caused by commensal organisms of bowel and perineum, particularly gram negative bacilli and enterococci[32].Researchers in Malaysia reported that Escherichia coli, andKlebsiella spp. are the main causative organisms [30].The patient's gender and the level of injury may affect the microbiology of organisms residing in the bladder. ForExample, Escherichia coli and Enterococcusspp, have been reported to cause more than two thirds of cases of UTI in female patients undergoing intermittent catheterization[33].In contrast,Klebsiellapneumonaie is one of the most common causes of UTI in hospitalized SCI patients[34],with particularly high prevalence of bacterial strains that exhibit strong 
type 1 fimbrialmediated adherence to uro-epithelial cells[35].In that regard E.coli strains that cause UTI in in adult SCI patients may exhibit more virulence factors(e.g.,hemolysis and-mannose-resistant hemaggultination of human erythrocytes) than strains asymptomatically reside in the bladder of SCI patients or the rectum of healthy volunteers[36].

Finding of polymicrobialbacteriuria is particularly problematic in SCI patients. Almost half of positive urine cultures in SCI grow more than one organism, and polymicrobial bacteriuria can be more prevalent in patients who have chronic indwelling ureteral catheters [37].Results of studies in otherwise healthy individuals indicating that short courses of oral antibiotics(a single large dose or a 3-day course) are efficacious in eradicating uncomplicated lower UTI should not be extrapolated to SCI population. A prospective study has shown a significantly better response to a 14-versus-day course of ciprofloxacin for treatment of UTI in SCI patients, but study excluded subjects with long term indwelling catheters [38].In patients with persistent infection, documented relapse of infection(by the same bacterial strain) or frequent reinfection(by different organisms), the urinary tract should be investigated for anatomic abnormalities(e.g., stone, abscess, stricture) and functional alteration(including vesicoureteral reflux and high residual volume of urine in bladder)[39].

\section{Bronchopneumonia}

Bronchopneumonia is less frequent than UTIs,pneumonia is the most common pulmonary complication in the immediate post injury period[40], and is particularly likely to occur in the first few months after cervical or high thoracic SCI and in quadriplegics and those at least 55 years of age[11].The relatively high mortality associated with pneumonia make it the leading cause of death caused by infection in this population[11].Pneumonia in acutely injured patients is associated with prolonged length of stay and escalated hospital costs[41].In patients who aspirate gastric contents, pneumonia is usually caused by gram negative and/or anaerobic bacteria. As in the case in able-bodied persons, bacterial community acquired pneumonia in SCI patients is mostly caused by Streptococcus pneumoniae,Haemophlis influenza, and Branhamellacatarrhalis.Staphylococcus aureus(mostly methicillin resistant[MRSA]) and Pseudomonas aeruginosa commonly cause pneumonia in mechanically ventilated patients and those with tracheostomy tubes.[42].

A number of noninfectious conditions can clinically mimic pneumonia.For example. atelectasis,like pneumonia, commonly occurs because of retained pulmonary secretions early after injury to the cervical or high thoracic cord. In such patients, with altered or absent sensation of chest pain and dyspnea and infective cough, the only clinical clues that suggest the diagnosis of pneumonia may include tachypnea, tachycardia fever and leukocytosis [26].Jackson and colleagues reported respiratory complication in $67 \%$ SCI patients with advance age and respiratory complications include atelectasis(36\%),pneumonia $(31 \%)$, and respiratory failure in $(22.6 \%)[43]$.

Another condition that can be clinically confused with pneumonia is pulmonary embolism, which occurs in 5\% ofSIPatients, frequently without an identifiable thrombotic source. Although pulmonary embolism can ordinarily be diagnosed by a ventilation perfusion lung scan, the observed defects on scanning may be uninterpretable in patients who also have atelectasis. In such cases, a pulmonary angiogram may be required for definitive diagnosis [26].

\section{Pressure Sores Infection}

Local factors that contribute to infection of pressure sores include breaks in the integrity of the skin barrier, pressure induced changes, and contamination from contagious dirty areas. Accordingly, pressure sores frequently become infected with staphylococci,streptococci or gram negative and/or anaerobic bacteria. Most pressures in SCI patients develop in areas adjacent to the ischium,sacrum,and and greater trochanter.In paralyzed who cannot directly visualize the ulcers, their history is usually incomplete and the infection is already advanced by the time they seek medical care. Patients with SCI are more likely than the general population to develop Fournier gangrene, the most frequent fearsome necrotizing fasciitis that affects the perineal and genital regions and usually results from polymicrobialinfection [44,54].

Because pressure sores are universally colonized, samples for culture should not be obtained unless infection is clinically evident. In patients with seemingly infected pressure ulcers, biopsy of deep tissue may constitute the most reliable means for determining the infectious cause. Cultures of swab specimens from the 
ulcer or the sinus tract are generally unreliable, and cultures of materials obtained by needle aspiration tend to overestimate the number of bacterial isolates [26].

A combined medical-surgical approach is often required to establish cure of the infection. The continuous application of negative pressure to deep ulcers by using a vacuum-assisted closure device (VAC) can enhance wound healing in selective patients by removing excessive edema, promoting granulation, and stimulating angiogenesis.[45].Although the sensitivity of nuclear scan for detecting soft tissue abscesses is generally very high, this test can also be positive in SCI patients who have an infected pressure sore without an associated abscess. Soft tissue abscess in association with an infected sore can be more accurately diagnosed by computed tomography (CT) or magnetic resonance imaging (MRI) than by nuclear scans[26].Joseph and associates reported pressure sores related infections in SCI patients, $42.9 \%$ in tetraplegia, and $19.6 \%$ in paraplegia. The incidence rate is reflective of the dedicated specialized care [46].

\section{Osteomyelitis in SCI Patients}

Most cases of osteomyelitis in SCI patients occur beneath pressure sores. Less common forms include prosthesis-related, postoperative. hematogenous, and vertebral osteomyelitis. In general, it is difficult to determine whether none beneath a decubitus ulcer is infected and, if infected which organism(s) is responsible. Cultures of a swab specimen from the ulcer are of little value in predicting the causative organisms of osteomyelitis. The definitive diagnosis of osteomyelitis beneath pressure sores requires histopathological examination of bone tissue[12].Because of the high frequency of bacterial colonization of fibrotic tissue adherent bone, cultures of bone specimens are positive in most patients in whom histopathologic examination of bone tissue is not compatible with osteomyelitis[12t].Most cases of osteomyelitis beneath pressure sores are caused by two or more bacterial species, including gram positive cocci(mainly S.aureus,streptococci) gram negative bacilli(including P.aeruginosaandEnterobacteriaceaespp)[12].

Clinical evaluation poorly predicts the presence of osteomyelitis beneath non-healing deep pressure sores. In particular, clinical information (duration of ulcer exposure, purulent drainage, fever), laboratory data (e.g., white blood cell count, erythrocyte sedimentation rate),and radiologic findings (plain roentgenograms and technetium bone scans) do not correlate well with likelihood of finding, histologic evidence of infection of bone. Nuclear scans are extremely sensitive but poorly specific for diagnosing osteomyelitis beneath pressure sores $[12,47]$.

\section{Infections due to multi- drug resistant organisms}

Multi drug resistant or multi-resistant bacteria have predilection to effect SCI units more than general nursing wards include MRSA (methicillin resistant Staphylococcus aureus),vancomycinresistantEnterococcus(VRE),gram- negative bacilli that produce extended spectrum $\beta$ lactamase(ESBL),and clostridium defficile. Roommate contacts of patients colonized or infected by any of these multi-resistant bacteria are at increased risk for acquiring those organisms[48].Of these multi-resistant organisms,MRSA is the most common; it is responsible for a large share of both hospital and community- acquired infections[49].Unlike MRSA, which could exist in almost every body organ, VRE is cultured mostly from the urine, particularly catheter-dependent patients. Although most episodes growth of VRE from urine cultures represent asymptomatic bacteremia and do not require antibiotic treatment,VRE colonization and residence in a longterm facility increase the risk for subsequent bacteremia[50].Catheter- dependent SCI patients are predisposed to develop urinary tract infection caused by ESBL-producing multi-resistant gram -negative bacilli, such as E.coli, andKlebsiella pneumoniae[51].Researchers in Malaysia reported that SCI patients with previous UTI, antibiotic usage during the past three months or history of hospitalization during past one -year were predisposed to develop infection with multi-resistant organisms[30].The frequent administration of antibiotics and relatively inadequate hygiene in SCI patients help explain the high risk of clinical infection by C.defficle.In patients with neurogenic bowel and defective sensation C.defficile associated gastrointestinal disease can remain clinically undetected until a catastrophe such as toxic megacolon or bowel perforation evolve[52].

\section{Bloodstream and Intra-abdominal Infection}

Infections of the urinary tract, pressure sores, lungs, and vascular access are the most common identifiable sources of bloodstream-bacteremia in patients with SCI[53].In bacteremia patients without an apparent source, an occult deep-seated abscess may be the culprit. Bacteremia associated with infections of the

DOI: $10.9790 / 0853-15078114120 \quad$ www.iosrjournals.org $\quad 118 \mid$ Page


urinary tract and long-term hemodialysis access are mostly caused by gram-negative bacilli, whereas Staphylococci are the most frequent isolates from the blood cultures in patients with infections of pressure sores or short-term vascular access [54].Because vascular catheter -related bacteremia is several-fold more likely to be caused by gram-negative bacteria in SCI patients than in the general population. Gram negative coverage should be considered when initiating empirical antibiotic therapy [55].

The most common intra-abdominal infections in SCI patients affect the gallbladder or present as abscesses.Cholellithiasis occurs more commonly in SCI patients than in the general population. Although most gallstones may remain asymptomatic, some may cause cholecystitis or pancreatitis. A ruptured viscus or, less commonly, a fistulous connection with a pressure sore can result in the formation of intra-abdominal abscess.Intra-abdominal infection may be misdiagnosed, particularly in patients with high cord lesions, because they frequently manifest with abdominal distention, diffuse spasm of abdominal wall musculature, and rigidity on palpation, but no localized abdominal pain or tenderness. Ultrasound examination or computed tomography of the abdomen should help establish the correct diagnosis. Nasogastric tube placement and impaired sinus drainage in the supine position predispose SCI patients to occult maxillary sinusitis [56].

\section{Conclusion}

Approximately $30 \%$ of the spinal cord injury (SCI) patients develop infection during hospitalization. SCI infections include urinary tract infection, pressure sores, osteomyelitis, bloodstream infection and intraabdominal infection. Pneumonia is associated with high mortality. Early diagnosis and treatment have better prognosis.

\section{References}

[1]. Hughes JT.The Edwin Smith Surgical Papyrus: An analysis of the first case reports of spinal cord injuries.Paraplegia.1988;26:7182.

[2]. National Spinal Cord Injury Statistical Center: Spinal cord facts and figures, June 2006 update.J Spinal Cord Med.2007;30:539-40.

[3]. Evans CT,LaVelaSL,WeaverFM,etal.Epidemiology of hospital acquired infection in vetrans with spinal cord injury and disorder.Infect Control HospEpidemiol. 2008;29: 234-42.

[4]. Peitzman,Fabian\&Rodes 2012;pp.288-89.

[5]. Sabharwal 2013;pp.53-54

[6]. Brown et al.2008;p.1132.

[7]. KabuS,GaoY,KwonBK,etal.Drugdelivery, cell- based therapies, and tissue engineering approaches for spinal cord injury.J ControlRelease.2015;219:141-54.

[8]. Frontera ,Silver\& Rizzo.2014;p.39.

[9]. Ibrahim A, Lee KY,KanooLL,etal.Epidemiology of spinal cord injury in Hospital Kuala Lumpur.Spine(Phila Pa 1976).2013;1;38(5):419-24.

[10]. Siroky MB. Pathogenesis of bacteriuria and infection in the spinal cord injury patient.Am J Med.2002;113(Suppl IA):67S-79S.

[11]. DeVivoMJ,KartusPL,StoverSl,et al. Cause of death for patients with spinal cord injury.Arch Intern Med.1989;149:1761-66.

[12]. DariouchiRO,LandonGC,KlimaM,etal.Oseteomyelitis associated with pressure sores.Arch Intern Med.1994;154:753-58.

[13]. Deck AJ,YangCC,Perinephric abscess in the neurologically impaired.Spinal Cord.2001;29:234-42.

[14]. GriverAR,PrinceRA,Darouiche RO.A simple method for administering vancomycin in the spinal cord-injured population. ArchPhys Med Rehabil.1997;78:459-162.

[15]. Gilman TM,BrunnmannSR,SegalJL.Comparison of population pharmacokinetic models for gentamicin in spinal cord injured and able bodied patients. Antimicrob Agent Chemother.1993;37:93-99.

[16]. LifshutzJ,Colohan A.A brief history of therapy for traumatic spinal cord injury.Neurosurg Focus.2004;16(1 E5):E5.18.doi:10.317/foc.2004.16.1.6

[17]. Holtz \& Levi 2010,pp.3-4.

[18]. Holtz\& Levi 2010,p.5

[19]. Holtz \&levi 2010,p.6.

[20]. Morganti-Kossmann,Raghupathi\& Maas 2012,p.229.

[21]. Fallah,Dance\& Bums 2012,p.235

[22]. Holtz \& Levi 2010,p.7.

[23]. Davies AJ,HayesKC,DekabanGA.Clinical correlates of elevated serum concentration of cytokines and autoantibodies in patients with spinal cord injury.Arch Phys Med Rehabil.2007;88:1384-93.

[24]. Wang TD,WangYH,HuangTS,etal.Circulation levels of markers of inflammation and endothelial activation are increased in with chronic spinal cord injury.JFormos Med Assoc.2007;106:919-28.

[25]. Darouiche R0.Spinal cord abscess Engl J Med.2006;355:2012-20.

[26]. Darouiche R0.Infections in Patients with Spinal Cord Injury.InMandell, DouglasandBennette'sPrinciples and Practice of InfectiousDiseases, $7^{\text {th }}$ ed.MandellGL,Bennett JE, Dolin R(editors).Churchill Livingstone Elsevier,2010.pp.3851-3856. 
[27]. Colachis S,0tis S. Occurrence of fever associated with thermoregulation dysfunction after acute spinal cord injury.Am $J$ PhysRehabil.!995;74:114-19.

[28]. SugarmanB.Fever in recently injured quadriplegic person.ArchPhys Med Rehabil.1982;63:639-40.

[29]. MirahmadiMK,BryneC,BartonC, etal.Prediction of creatinine clearance from serum creatinine in spinal cord injury patients.Paraplegia..1983;21:23-29.

[30]. ToganT,AzapOK,Durakan,E,etal.ThePrevalence,etiologic agents and risk factors for Urinary tract infection among Spinal cord injury patients.JundishapurJ Microbiol.2014;7(1):e8905.

[31]. ShekellePG,MortonSC,ClarkKK, etal.Systematic review of risk factors for urinary tract infection in adults and spinal cord dysfunction... S Spinal Cord Med.1999;22:258-72.

[32]. Waites KB,CanuppKC,DeVivoMJ.Microbiology of the urethra and perineum and its relationship with to bacteriuria in community residing men with spinal cord injury.J Spinal Cord Med.2004;27:448-452.

[33]. Bennett CJ,YoungMN,DarringtonH.Differences in urinary tract infection in male and female spinal cord injury patients on intermittent cathetherization.Parapelagia.1995;33:69-72.

[34]. Darouiche R0,Cadle R,ZenonG,etal.Pregression from asymptomatic to symptomatic urinary tract infection in patients with SCI:A preliminary study.J Am Paraplegia Soc.1993;16:221-26.

[35]. KilKS,Darouiche R0,Hull RA,etal.Identification of a Klebsiella pneumoniae strain associated with nosocomial urinary tract infection.JClin Microbiol.1997;35:2370-74.

[36]. Hull RA,RudyDC,WieserJE,etal.Virulence factors of Escherichia coli isolates from patient with symptomatic and asymptomatic bacteriuria and neuropathic bladder due to spinal cord and brain injuries.JClin Microbiol.1998;36:115-17.

[37]. Darouiche R0,PriebM,ClarridgeJE.Limited vs full microbiological investigation for the management of symptomatic poly microbial urinary tract infection in adult spinal cord-injured patients. Spinal Cord.1997;35:534-39.

[38]. Dow G,RaoR,HardingG,etal.A prospective randomized trial of 3 or 14 days of ciprofloxacine treatment for acute urinary tract infection in patients with spinal cord injury.Clin Infect Dis.2004;39:658-64.

[39]. Joshi A,Darouiche R0.Regression of pyuria during treatment of symptomatic urinary tract infection in patients with spinal cord injury.Spinal Cord.1996;34:742-44.

[40]. Burns SP.Acute respiratory infections in persons with spinal cord injury.Phys Med RehabilClin North Am.2004;42:450-58.

[41]. Winslow C,BodeKK,FeltonD,etal.Impact of respiratory complication on length of stay and hospital costs in acute cervical spine injury.Chest.2002;121:1548-54.

[42]. Chang HT,EvansCT,WaeverFM,etal.Etiology and outcomes of veterans with spinal cord injury and disorders hospitalized with community-acquired pneumonia.Arch Phys Med Rehabil.2005;86:262-67.

[43]. Jackson AB,GroomesTE.Incidence of respiratory complications following spinal cord injury .Arch Phys Med Rehabil.1994;75:2705 .

[44]. NambiarPK,LanderS,MidhaM,etal.Fournier gangrene spinal cord injury:A case report.J Spinal Cord Med.2005;28:212-24.

[45]. Ford CN,ReinhandER, YehD, etal.Interim analysis of a prospective randomized trial of vacuum-assisted closure versus the health point system in the management of pressure ulcers. Ann Plast Surg.2002;49:55-61.

[46]. Joseph LH,Ismail $0 \mathrm{H}$, NaickerAS, etal.Three- year study of spinal cord injury outcomes and related secondaty complications in a tertiary centre-a retrospective analysis.Arch Med Sci..2009;5:190-4.

[47]. MelkunET,Lewis VL Jr, Evaluation of (111) indium labeled autologous leukocyte for the diagnosis of chronic osteomyelitis in patients with grade IV pressure ulcers, as compared with a standard diagnostic protocol.AmPlast Surg.2005;54:633-36.

[48]. Zhou Q,MooreC,EdenS,etal.Mount Sinai Hospital Infection Control Team. Factors associated with acquisition of vancomycinresistant enterococci(VRE) in roommate contacts of patients colonized or infected with VRE in a tertiary care hospital.InfectControl Hosp Epidemiol.2008;29:398- 403.

[49]. KappelC,WidmerA,GengV,et al. Successful control of methicillin -resistant Staphylococcus aureus in spinal cord injury center.: A year prospective study including molecular typing.Spinal Cord.2008;46:438-44.

[50]. Olivier CN,BlakeRK,SteedLL,etal.Risk of vancomycin resistant enterococcus(VRE) bloodstream infection among patients colonized with VRE.Infect Control Hosp Epidemiol.2008;29:404-409.

[51]. Apisarnthanark A, Bailey TC,FraserVJ.Duration of stool colonization in patients infected with extended -spectrum $\beta$ lactamase producing Escherichia coli and Klebsiellapneumoniae.Clin Infect Dis.2008;46:1322-23.

[52]. MarciniakC,ChenD,SteinAC,etal.Prevalence of Clostridium defficile colonization at admission to rehabilitation.Arch Phys MedRehabil.2006;87:1006-1090.

[53]. MylotteJm,GrahamR,KablerL,etal.Epidemiology of nosocomial infection and resistant organism in patients admitted for the first time to an acute rehabilitation unit.Clin Infect Dis.2000;30:425-32.

[54]. Wall BM,MangoldT,HuchKM,etal.Bacteremia in the chronic spinal cord injury populations::Risk Factors for mortality. $J$ SpinalCord Med.2003;26:248-53.

[55]. Hussain R,CevallosME,Darouiche R0,et al.Gram-negative intravascular-catheter related bacteremia in patients with spinal cord injury.Arch Phys Med Rehabil. 2008;89:339-42.

[56]. Lew HL,HanJ,RobinsinLR,etal.Occult maxillary sinusitis a cause of fever in tetraplegic:2 case reports. Arch Phys MedRehabil.2002;83:430-32. 